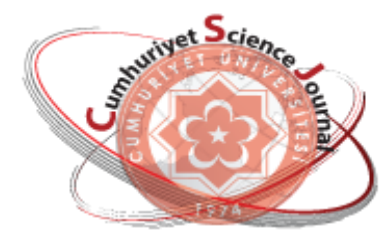

e-ISSN: $2587-246 X$

ISSN: $2587-2680$

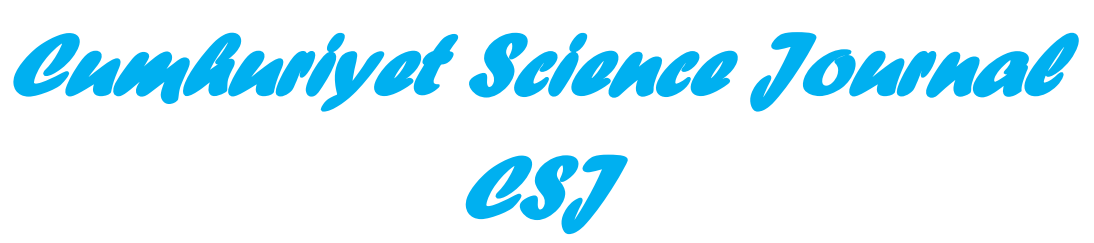

Cumhuriyet Sci. J., Vol.39-2(2018) 303-313

\title{
Some Results On Quaternion 3-Space
}

\author{
Atilla AKPINAR*, Fatma OZEN ERDOGAN \\ University of Uludag, Faculty of Arts and Science, Department of Mathematics, Bursa, TURKEY \\ Received: 10.04.2017; Accepted: 10.04.2018 \\ http://dx.doi.org/10.17776/csj.434228
}

\begin{abstract}
In this paper, the set $\mathbf{J}^{\prime}=\mathbf{H}\left(\mathrm{Q}_{4}, \mathrm{~J} \gamma\right)$ of 4 by 4 matrices, with entries in a quaternion F-algebra $\mathrm{Q}$, that are symmetric with respect to the canonical involution $\mathbf{J} \gamma$ is studied. $\mathbf{J}^{\prime}$ is also the special Jordan matrix algebra and some results related to points and lines of the quaternion 3-space $\mathbf{P}\left(\mathbf{J}^{\prime}\right)$ defined by the algebra are introduced. Finally, by taking dual ring $\mathbf{Q}:=\mathrm{Q}+\mathrm{Q} \varepsilon\left(\varepsilon \notin \mathrm{Q}, \varepsilon^{2}=0\right)$ instead of $\mathrm{Q}$, the obtained results are carried to a more general state.
\end{abstract}

2010 Mathematics Subject Classification: 51C05; 17C55; 05B25

Keywords: Special Jordan matrix algebra, quaternion, quaternion 3-space.

\section{Kuaterniyon 3-Uzay Üzerine Bazı Sonuçlar}

Özet: $\mathrm{Bu}$ makalede, girdileri bir $\mathrm{Q}$ kuaterniyon F-cebirinden alınan ve $\mathrm{J} \gamma$ kanonik involusyonuna göre simetrik olan $4 \times 4$ boyutlu matrislerin oluşturduğu $\mathbf{J}^{\prime}=\mathbf{H}\left(Q_{4}, \mathrm{~J} \gamma\right)$ kümesi ile çalışılmıştır. Bu $\mathbf{J}^{\prime}$ kümesi aynı zamanda bir özel Jordan matris cebiridir ve bu cebir ile tanımlanan $\mathbf{P}\left(\mathbf{J}^{\prime}\right)$ kuaterniyon 3-uzayın noktalar ve doğruları ile ilgili bazı sonuçlar sunulmuştur. Son olarak, $\mathbf{Q}$ yerine $\mathbf{Q}:=\mathrm{Q}+\mathrm{Q} \varepsilon\left(\varepsilon \notin \mathrm{Q}, \varepsilon^{2}=0\right)$ dual halkası alınarak elde edilen sonuçlar daha genel bir duruma taşınmıştır.

Anahtar Kelimeler: Özel Jordan matris cebiri, kuaterniyon, kuaterniyon 3-uzay

\section{INTRODUCTION and PRELIMINARIES}

In [5], Faulkner deals with $\mathbf{J}=\mathbf{H}\left(\mathbf{O}_{3}, J \gamma\right)$, the set of 3 by 3 matrices with entries in an octonion algebra $\mathbf{O}$ defined over a field $\mathrm{F}$, that are symmetric with respect to the canonical involution $\mathrm{J} \gamma: \mathrm{X} \rightarrow \gamma^{-1} \overline{\mathrm{X}}^{\mathrm{t}} \gamma$ where the $\gamma_{i}$ are non-zero elements of $F$ and $\gamma:=\operatorname{diag}\left\{\gamma_{1}, \gamma_{2}, \gamma_{3}\right\}$. Hence, any element $X$ of $\mathbf{J}$ is of the form

$\mathrm{X}=\left(\begin{array}{ccc}\alpha_{1} & \gamma_{2} \mathrm{a}_{3} & \gamma_{3} \overline{\mathrm{a}}_{2} \\ \gamma_{1} \overline{\mathrm{a}}_{3} & \alpha_{2} & \gamma_{3} \mathrm{a}_{1} \\ \gamma_{1} \mathrm{a}_{2} & \gamma_{2} \overline{\mathrm{a}}_{1} & \alpha_{3}\end{array}\right)$ for $\alpha_{\mathrm{i}} \in \mathrm{F}$ and $\mathrm{a}_{\mathrm{i}} \in \mathbf{O}$

If it is defined a cubic form $N$ such that $N(X):=\operatorname{det} X$, a quadratic mapping $X \rightarrow X^{\#}:=$ adjoint of $X$, and a basepoint $\mathrm{C}:=\mathrm{I}_{3}$ on $\mathbf{J}$ are defined, then the triple $(\mathbf{J}, \mathrm{N}, \mathrm{C})$ is a quadratic (exceptional) Jordan algebra 
under the operator $U_{X} Y=T(X, Y) X-2\left(X^{\#} \times Y\right) \quad[9]$. Then, for $X=\left(\begin{array}{ccc}\alpha_{1} & \gamma_{2} \mathrm{a}_{3} & \gamma_{3} \bar{a}_{2} \\ \gamma_{1} \bar{a}_{3} & \alpha_{2} & \gamma_{3} \mathrm{a}_{1} \\ \gamma_{1} \mathrm{a}_{2} & \gamma_{2} \overline{\mathrm{a}}_{1} & \alpha_{3}\end{array}\right)$ and $\mathrm{Y}=\left(\begin{array}{ccc}\beta_{1} & \gamma_{2} \mathrm{~b}_{3} & \gamma_{3} \overline{\mathrm{b}}_{2} \\ \gamma_{1} \overline{\mathrm{b}}_{3} & \beta_{2} & \gamma_{3} \mathrm{~b}_{1} \\ \gamma_{1} \mathrm{~b}_{2} & \gamma_{2} \overline{\mathrm{b}}_{1} & \beta_{3}\end{array}\right) \in \mathbf{J}$, we can give the similar results to those given in $[6,9]$ :

$\mathrm{N}(\mathrm{X})=\alpha_{1} \alpha_{2} \alpha_{3}-\alpha_{1} \gamma_{2} \gamma_{3} \mathrm{n}\left(\mathrm{a}_{1}\right)-\alpha_{2} \gamma_{3} \gamma_{1} \mathrm{n}\left(\mathrm{a}_{2}\right)-\alpha_{3} \gamma_{1} \gamma_{2} \mathrm{n}\left(\mathrm{a}_{3}\right)+\gamma_{1} \gamma_{2} \gamma_{3} 2 \mathrm{t}\left(\left(\mathrm{a}_{1} \mathrm{a}_{2}\right) \mathrm{a}_{3}\right)$

$X^{\#}=\left(X_{i j}\right)_{3 \times 3}$ for $X_{i i}=\alpha_{j} \alpha_{k}-\gamma_{j} \gamma_{k} n\left(a_{i}\right), X_{i j}=\gamma_{i} \gamma_{k} a_{i} a_{j}-\gamma_{i} \alpha_{k} \bar{a}_{k}$ and $X_{j i}=\overline{X_{i j}}$,

$\mathrm{X} \times \mathrm{Y}=\left(\mathrm{z}_{\mathrm{ij}}\right)_{3 \times 3}$ for $\left\{\begin{array}{l}\mathrm{z}_{\mathrm{ii}}=(1 / 2)\left[\alpha_{\mathrm{j}} \beta_{\mathrm{k}}+\beta_{\mathrm{j}} \alpha_{\mathrm{k}}-2 \gamma_{\mathrm{j}} \gamma_{\mathrm{k}} \mathrm{n}\left(\mathrm{a}_{\mathrm{i}}, \mathrm{b}_{\mathrm{i}}\right)\right] \\ \mathrm{z}_{\mathrm{ii}}=(1 / 2)\left(\gamma_{\mathrm{j}}\left[\gamma_{\mathrm{k}} \overline{\left(\mathrm{a}_{\mathrm{i}} \mathrm{b}_{\mathrm{j}}+\mathrm{b}_{\mathrm{i}} \mathrm{a}_{\mathrm{j}}\right)}-\left(\alpha_{\mathrm{k}} \mathrm{b}_{\mathrm{k}}+\beta_{\mathrm{k}} \mathrm{a}_{\mathrm{k}}\right)\right]\right), \mathrm{z}_{\mathrm{ji}}=\overline{\mathrm{z}}_{\mathrm{ij}},\end{array}\right.$

$\mathrm{T}(\mathrm{X}, \mathrm{Y})=\alpha_{1} \beta_{1}+\alpha_{2} \beta_{2}+\alpha_{3} \beta_{3}+2 \gamma_{2} \gamma_{3} \mathrm{n}\left(\mathrm{a}_{1}, \mathrm{~b}_{1}\right)+2 \gamma_{3} \gamma_{1} \mathrm{n}\left(\mathrm{a}_{2}, \mathrm{~b}_{2}\right)+2 \gamma_{1} \gamma_{2} \mathrm{n}\left(\mathrm{a}_{3}, \mathrm{~b}_{3}\right)$,

where $(\mathrm{i}, \mathrm{j}, \mathrm{k})$ is a cyclic permutation of $(1,2,3), \mathrm{n}$ (defined by $\mathrm{n}(\mathrm{x}):=\mathrm{x} \overline{\mathrm{x}}$ ) is the norm (quadratic) form over $\mathbf{O}, \mathrm{t}$ (defined by $\mathrm{t}(\mathrm{x}):=(1 / 2)(\mathrm{x}+\overline{\mathrm{x}})$ ) is the trace (linear) form over $\mathbf{O}$ and finally $\mathrm{n}(\mathrm{x}, \mathrm{y})$ (defined by $\mathrm{n}(\mathrm{x}, \mathrm{y}):=(1 / 2)[\mathrm{n}(\mathrm{x}+\mathrm{y})-\mathrm{n}(\mathrm{x})-\mathrm{n}(\mathrm{y})])$ is symmetric bilinear norm w.r.t. $\mathrm{n}$.

Let $\Pi$ denote the set of elements of rank 1 in $\mathbf{J}$. Then,

$\Pi=\left\{X \mid X \in J-\{0\}\right.$ and $\left.X \times X=X^{\#}=0\right\}$.

Note that, if $\mathrm{X} \in \Pi$ and $\alpha$ is a non-zero element in $\mathrm{F}$, then $\alpha \mathrm{X} \in \Pi$. For $\mathrm{X} \in \Pi$, let $\mathrm{X}_{*}$ and $\mathrm{X}^{*}$ be two copies of the set $\{\alpha \mathrm{X} \mid \alpha \in \mathrm{F}-\{0\}\}$.

Now, we are ready to give the definition of an octonion plane $\mathbf{P}(\mathbf{J})$ from [5, Chapter 3].

The octonion plane $\mathbf{P}(\mathbf{J})=(\mathbf{P}, \mathbf{L}, \mid, \sqcup)$ consists of the incidence structure $(\mathbf{P}, \mathbf{L}, \mid)$ (points, lines, and incidence), and the connection relation is defined as follows:

$\mathbf{P}=\left\{\mathrm{X}_{*} \mid \mathrm{X} \in \Pi\right\}, \mathbf{L}=\left\{\mathrm{X}^{*} \mid \mathrm{X} \in \Pi\right\}$,

$\mathrm{X}_{*} \mid \mathrm{Y}^{*}, \mathrm{X}_{*}$ is on $\mathrm{Y}^{*}$, if $\mathrm{V}_{\mathrm{Y}, \mathrm{X}}=0$, that is, $\mathrm{V}_{\mathrm{Y}, \mathrm{X}}=:\{1 \mathrm{XY}\}=\{\mathrm{X} 1 \mathrm{Y}\}=\{\mathrm{XY} 1\}=\mathrm{X} \cdot \mathrm{Y}=0$ where $\mathrm{X} \cdot \mathrm{Y}=(1 / 2)(\mathrm{XY}+\mathrm{YX})($ Jordan multiplication),

$\mathrm{X}_{*} \sqcup \mathrm{Y}_{*}, \mathrm{X}_{*}$ is connected to $\mathrm{Y}_{*}$ if $\mathrm{X} \times \mathrm{Y}=0$,

$\mathrm{X}^{*} \sqcup \mathrm{Y}^{*}, \mathrm{X}^{*}$ is connected to $\mathrm{Y}^{*}$ if $\mathrm{X} \times \mathrm{Y}=0$,

$\mathrm{X}_{*} \sqcup \mathrm{Y}^{*}, \mathrm{X}_{*}$ is connected (or near) to $\mathrm{Y}^{*}$ if $\mathrm{T}(\mathrm{X}, \mathrm{Y})=0$.

In [7, Chapter III.2, Theorem 1], Jacobson showed that the fact that $\left(\mathbf{D}_{\mathrm{n}}, \mathrm{J} \gamma\right)$ is a Jordan algebra implies that $\mathbf{D}$ is associative if $n \geq 4$ but alternative with its symmetric elements in the nucleus if $n=3$. Therefore, in the case of $\mathrm{n} \geq 4$ we can study with the elements of a quaternion algebra, which is associative (but not commutative) and moreover the Jordan matrix algebra $\left(\mathbf{D}_{\mathrm{n}}, J \gamma\right)$ is necessarily special (that is, not exceptional) since $\mathbf{D}$ is associative [7, p.138]. 
Let $\mathrm{F}$ be a field and let $\mathrm{Q}=\left\{\mathrm{r}_{0}+\mathrm{r}_{1} i_{1}+\mathrm{r}_{2} i_{2}+\mathrm{r}_{3} i_{3} \mid \mathrm{r}_{\mathrm{i}} \in \mathrm{F}\right\}$ be a quaternion division F-algebra. From now on, we assume that the characteristic of $\mathrm{F}$ is different from 2. We denote the anti-involution over $\mathrm{Q}$ by $\mathrm{j}$ $(\mathrm{j}(\mathrm{x}):=\mathrm{x})$, the norm (quadratic) form over $\mathrm{Q}$ by $\mathrm{n}(\mathrm{n}(\mathrm{x}):=\mathrm{x} \overline{\mathrm{x}} \in \mathrm{F})$, and the trace (linear) form over $\mathrm{Q}$ by $\mathrm{t}$ $(t(x):=(1 / 2)(x+\bar{x}) \in F)$. In this case, $x=r_{0}-r_{1} i_{1}-r_{2} i_{2}-r_{3} i_{3}, n(x)=r_{0}{ }^{2}-c_{1} r_{1}{ }^{2}-c_{2} r_{2}{ }^{2}+c_{1} c_{2} r_{3}{ }^{2}$ where $c_{1}, c_{2}$ are nonzero elements in the multiplication table [8, p.448] and $t(x)=r_{0}$ for any $x=r_{0}+r_{1} i_{1}+r_{2} i_{2}+r_{3} i_{3} \in Q$. For example, for $\mathrm{F}=\mathbb{R}$ and $\mathrm{c}_{1}=\mathrm{c}_{2}=-1$ we have Hamilton's quaternion (division) algebra and so we reach the result: $\mathrm{n}(\mathrm{x})=\mathrm{r}_{0}^{2}+\mathrm{r}_{1}^{2}+\mathrm{r}_{2}^{2}+\mathrm{r}_{3}{ }^{2}=0 \Leftrightarrow \mathrm{x}=0$.

$\mathbf{J}^{\prime}=\mathbf{H}\left(\mathrm{Q}_{4}, \mathrm{~J} \gamma\right)$, the set of 4 by 4 matrices, with entries in an quaternion division F-algebra, that are symmetric with respect to the canonical involution $J \gamma: X \rightarrow \gamma^{-1} \bar{X}^{t} \gamma$ where the $\gamma_{i}$ are non-zero elements of F and $\gamma:=\operatorname{diag}\left\{\gamma_{1}, \gamma_{2}, \gamma_{3}, \gamma_{4}\right\}$. Hence, any element $X$ of $\mathbf{J}^{\prime}$ is of the form

$$
\mathrm{X}=\left[\mathrm{X}_{\mathrm{ij}}\right]=\left(\begin{array}{cccc}
\alpha_{1} & \gamma_{2} \mathrm{a}_{12} & \gamma_{3} \overline{\mathrm{a}}_{13} & \gamma_{4} \mathrm{a}_{14} \\
\gamma_{1} \overline{\mathrm{a}}_{12} & \alpha_{2} & \gamma_{3} \mathrm{a}_{23} & \gamma_{4} \overline{\mathrm{a}}_{24} \\
\gamma_{1} \mathrm{a}_{13} & \gamma_{2} \overline{\mathrm{a}}_{23} & \alpha_{3} & \gamma_{4} \mathrm{a}_{34} \\
\gamma_{1} \overline{\mathrm{a}}_{14} & \gamma_{2} \mathrm{a}_{24} & \gamma_{3} \overline{\mathrm{a}}_{34} & \alpha_{4}
\end{array}\right) \text { for } \alpha_{\mathrm{i}} \in \mathrm{F} \text { and } \mathrm{a}_{\mathrm{ij}} \in \mathrm{Q}
$$

If we take a quartic (fourth degree) form $\mathrm{N}$ such that $\mathrm{N}(\mathrm{X}):=\operatorname{det} \mathrm{X}$, a cubic mapping $\mathrm{X} \rightarrow \mathrm{X}^{\#}:=$ adjoint of $\mathrm{X}$, and a basepoint $\mathrm{C}:=\mathrm{I}_{4}$ on $\mathbf{J}$, then: it is clear that

$$
\begin{aligned}
\mathrm{T}(\mathrm{X}, \mathrm{Y})= & \alpha_{1} \beta_{1}+\alpha_{2} \beta_{2}+\alpha_{3} \beta_{3}+\alpha_{4} \beta_{4}+2 \gamma_{1} \gamma_{2} \mathrm{n}\left(\mathrm{a}_{12}, \mathrm{~b}_{12}\right)+2 \gamma_{1} \gamma_{3} \mathrm{n}\left(\mathrm{a}_{13}, \mathrm{~b}_{13}\right)+2 \gamma_{1} \gamma_{4} \mathrm{n}\left(\mathrm{a}_{14}, \mathrm{~b}_{14}\right) \\
& +2 \gamma_{2} \gamma_{3} \mathrm{n}\left(\mathrm{a}_{23}, \mathrm{~b}_{23}\right)+2 \gamma_{2} \gamma_{4} \mathrm{n}\left(\mathrm{a}_{24}, \mathrm{~b}_{24}\right)+2 \gamma_{3} \gamma_{4} \mathrm{n}\left(\mathrm{a}_{34}, \mathrm{~b}_{34}\right),
\end{aligned}
$$

as $T(X, Y):=T(X \cdot Y)=\operatorname{trace}(X \cdot Y)$. Besides, note that $X \times Y$ must be equal to $(1 / 6)\left[(X+Y)^{\#}-X^{\#}-Y^{\#}\right]$ and specifically, $X \times X=X^{\#}$ as in the case of $n=3$.

Now, from [1], we can give some informations about the quaternion (but, not dual) 3-space $\mathbf{P}\left(\mathbf{J}^{\prime}\right)=(\mathbf{P}, \mathbf{L}, \mid, \sqcup)$ where $\mathbf{J}^{\prime}$ is the 28-dimensional special Jordan matrix algebra. Then, the set of points $\mathbf{P}$ consists of the following four classes (which we call as points of types 1,2,3 and 4, respectively):

$$
\begin{aligned}
& \left\{P_{1}=\left(\begin{array}{cccc}
1 & \gamma_{1}^{-1} \gamma_{2} \bar{x}_{2} & \gamma_{1}^{-1} \gamma_{3} \bar{x}_{3} & \gamma_{1}^{-1} \gamma_{4} \bar{x}_{4} \\
x_{2} & \gamma_{1}^{-1} \gamma_{2} n\left(x_{2}\right) & \gamma_{1}^{-1} \gamma_{3} x_{2} \bar{x}_{3} & \gamma_{1}^{-1} \gamma_{4} x_{2} \bar{x}_{4} \\
x_{3} & \gamma_{1}^{-1} \gamma_{2} x_{3} \bar{x}_{2} & \gamma_{1}^{-1} \gamma_{3} n\left(x_{3}\right) & \gamma_{1}^{-1} \gamma_{4} x_{3} \bar{x}_{4} \\
x_{4} & \gamma_{1}^{-1} \gamma_{2} x_{4} \bar{x}_{2} & \gamma_{1}^{-1} \gamma_{3} x_{4} \bar{x}_{3} & \gamma_{1}^{-1} \gamma_{4} n\left(x_{4}\right)
\end{array}\right)=:\left(\begin{array}{c}
1 \\
x_{2} \\
x_{3} \\
x_{4}
\end{array}\right)^{t} \mid x_{i} \in Q\right\} \cup \\
& \left\{P_{2}=\left(\begin{array}{cccc}
0 & 0 & 0 & 0 \\
0 & 1 & \gamma_{2}^{-1} \gamma_{3} \bar{x}_{3} & \gamma_{2}^{-1} \gamma_{4} \bar{x}_{4} \\
0 & x_{3} & \gamma_{2}^{-1} \gamma_{3} n\left(x_{3}\right) & \gamma_{2}^{-1} \gamma_{4} x_{3} \bar{x}_{4} \\
0 & x_{4} & \gamma_{2}{ }^{-1} \gamma_{3} x_{4} \bar{x}_{3} & \gamma_{2}{ }^{-1} \gamma_{4} n\left(x_{4}\right)
\end{array}\right)=:\left(\begin{array}{c}
0 \\
1 \\
x_{3} \\
x_{4}
\end{array}\right)^{t} \mid x_{3}, x_{4} \in Q\right\} \cup \\
& \left\{\mathrm{P}_{3}=\left(\begin{array}{cccc}
0 & 0 & 0 & 0 \\
0 & 0 & 0 & 0 \\
0 & 0 & 1 & \gamma_{3}{ }^{-1} \gamma_{4} \overline{\mathrm{x}}_{4} \\
0 & 0 & \mathrm{x}_{4} & \gamma_{3}{ }^{-1} \gamma_{4} \mathrm{n}\left(\mathrm{x}_{4}\right)
\end{array}\right)=:\left(\begin{array}{c}
0 \\
0 \\
1 \\
\mathrm{x}_{4}
\end{array}\right)^{\mathrm{t}} \mid \mathrm{x}_{4} \in \mathrm{Q}\right\} \cup\left\{\mathrm{P}_{4}=\left(\begin{array}{cccc}
0 & 0 & 0 & 0 \\
0 & 0 & 0 & 0 \\
0 & 0 & 0 & 0 \\
0 & 0 & 0 & 1
\end{array}\right)=:\left(\begin{array}{l}
0 \\
0 \\
0 \\
1
\end{array}\right)^{\mathrm{t}}\right\},
\end{aligned}
$$


the set of lines $\mathbf{L}$ consists of the following four classes (which we call as lines of types 1,2,3 and 4, respectively):

$$
\begin{aligned}
& \left\{l_{1}=\left[\begin{array}{cccc}
1 & 0 & 0 & 0 \\
0 & 0 & 0 & 0 \\
0 & 0 & 0 & 0 \\
0 & 0 & 0 & 0
\end{array}\right]=:\left[\begin{array}{l}
1 \\
0 \\
0 \\
0
\end{array}\right]^{t}\right\} \cup \\
& \left\{l_{2}=\left[\begin{array}{cccc}
\gamma_{1}{ }^{-1} \gamma_{2} n\left(m_{1}\right) & -\gamma_{1}{ }^{-1} \gamma_{2} \bar{m}_{1} & 0 & 0 \\
-\mathrm{m}_{1} & 1 & 0 & 0 \\
0 & 0 & 0 & 0 \\
0 & 0 & 0 & 0
\end{array}\right]=:\left[\begin{array}{c}
\mathrm{m}_{1} \\
1 \\
0 \\
0
\end{array}\right]^{\mathrm{t}} \mid \mathrm{m}_{1} \in \mathrm{Q}\right\} \cup
\end{aligned}
$$

$\left\{1_{3}=\left[\begin{array}{cccc}\gamma_{1}^{-1} \gamma_{3} n\left(m_{1}\right) & \gamma_{1}{ }^{-1} \gamma_{3} \bar{m}_{1} m_{2} & -\gamma_{1}^{-1} \gamma_{3} \bar{m}_{1} & 0 \\ \gamma_{2}{ }^{-1} \gamma_{3} \bar{m}_{2} m_{1} & \gamma_{2}{ }^{-1} \gamma_{3} n\left(m_{2}\right) & -\gamma_{2}^{-1} \gamma_{3} \bar{m}_{2} & 0 \\ -m_{1} & -m_{2} & 1 & 0 \\ 0 & 0 & 0 & 0\end{array}\right]=:\left[\begin{array}{c}m_{1} \\ m_{2} \\ 1 \\ 0\end{array}\right]^{t} \mid m_{1}, m_{2} \in Q\right\} U$

$\left\{1_{4}=\left[\begin{array}{cccc}\gamma_{1}^{-1} \gamma_{4} \mathrm{n}\left(\mathrm{m}_{1}\right) & \gamma_{1}{ }^{-1} \gamma_{4} \overline{\mathrm{m}}_{1} \mathrm{~m}_{2} & \gamma_{1}{ }^{-1} \gamma_{4} \overline{\mathrm{m}}_{1} \mathrm{~m}_{3} & -\gamma_{1}^{-1} \gamma_{4} \overline{\mathrm{m}}_{1} \\ \gamma_{2}{ }^{-1} \gamma_{4} \overline{\mathrm{m}}_{2} \mathrm{~m}_{1} & \gamma_{2}{ }^{-1} \gamma_{4} \mathrm{n}\left(\mathrm{m}_{2}\right) & \gamma_{2}{ }^{-1} \gamma_{4} \overline{\mathrm{m}}_{2} \mathrm{~m}_{3} & -\gamma_{2}{ }^{-1} \gamma_{4} \overline{\mathrm{m}}_{2} \\ \gamma_{3}{ }^{-1} \gamma_{4} \overline{\mathrm{m}}_{3} \mathrm{~m}_{1} & \gamma_{3}{ }^{-1} \gamma_{4} \overline{\mathrm{m}}_{3} \mathrm{~m}_{2} & \gamma_{3}{ }^{-1} \gamma_{4} \mathrm{n}\left(\mathrm{m}_{3}\right) & -\gamma_{3}{ }^{-1} \gamma_{4} \overline{\mathrm{m}}_{3} \\ -\mathrm{m}_{1} & -\mathrm{m}_{2} & -\mathrm{m}_{3} & 1\end{array}\right]=:\left[\begin{array}{l}\mathrm{m}_{1} \\ \mathrm{~m}_{2} \\ \mathrm{~m}_{3} \\ 1\end{array}\right]^{\mathrm{t}} \mid \mathrm{m}_{\mathrm{i}} \in \mathrm{Q}\right\}$

The incidence relation |, equivalent to $\mathrm{X} \cdot \mathrm{Y}=0$, is defined as follows:

$$
\begin{aligned}
& {[1,0,0,0]=\left\{\left(0,1, \mathrm{y}_{3}, \mathrm{y}_{4}\right) \mid \mathrm{y}_{3}, \mathrm{y}_{4} \in \mathrm{Q}\right\} \cup\left\{\left(0,0,1, \mathrm{z}_{4}\right) \mid \mathrm{z}_{4} \in \mathrm{Q}\right\} \cup\{(0,0,0,1)\},} \\
& {\left[1_{1}, 1,0,0\right]=\left\{\left(1,1_{1}, \mathrm{x}_{3}, \mathrm{x}_{4}\right) \mid \mathrm{x}_{3}, \mathrm{x}_{4} \in \mathrm{Q}\right\} \cup\left\{\left(0,0,1, \mathrm{z}_{4}\right) \mid \mathrm{z}_{4} \in \mathrm{Q}\right\} \cup\{(0,0,0,1)\},} \\
& {\left[\mathrm{m}_{1}, \mathrm{~m}_{2}, 1,0\right]=\left\{\left(1, \mathrm{x}_{2}, \mathrm{~m}_{1}+\mathrm{m}_{2} \mathrm{x}_{2}, \mathrm{x}_{4}\right) \mid \mathrm{x}_{2}, \mathrm{x}_{4} \in \mathrm{Q}\right\} \cup\left\{\left(0,1, \mathrm{~m}_{2}, \mathrm{y}_{4}\right) \mid \mathrm{y}_{4} \in \mathrm{Q}\right\} \cup\{(0,0,0,1)\},} \\
& {\left[\mathrm{n}_{1}, \mathrm{n}_{2}, \mathrm{n}_{3}, 1\right]=\left\{\left(1, \mathrm{x}_{2}, \mathrm{x}_{3}, \mathrm{n}_{1}+\mathrm{n}_{2} \mathrm{x}_{2}+\mathrm{n}_{3} \mathrm{x}_{3},\right) \mid \mathrm{x}_{2}, \mathrm{x}_{3} \in \mathrm{Q}\right\} \cup\left\{\left(0,1, \mathrm{y}_{3}, \mathrm{n}_{2}+\mathrm{n}_{3} \mathrm{y}_{3},\right) \mid \mathrm{y}_{3} \in \mathrm{Q}\right\} \cup} \\
& \left\{\left(0,0,1, \mathrm{n}_{3}\right)\right\} .
\end{aligned}
$$

Finally by the relation equivalent to the connection relation $\sqcup$ given by $X \times Y=0$ in the case $n=3$ (see [2] for this equivalence), we can define the connection relation $\sqcup$ in this space as follows:

$$
\begin{aligned}
& \left(\mathrm{x}_{1}, \mathrm{x}_{2}, \mathrm{x}_{3}, \mathrm{x}_{4}\right) \sqcup\left(\mathrm{y}_{1}, \mathrm{y}_{2}, \mathrm{y}_{3}, \mathrm{y}_{4}\right) \Leftrightarrow \mathrm{x}_{\mathrm{i}}-\mathrm{y}_{\mathrm{i}}=0 \text { for } \mathrm{i}=1,2,3,4, \\
& {\left[\mathrm{k}_{1}, \mathrm{k}_{2}, \mathrm{k}_{3}, \mathrm{k}_{4}\right] \sqcup\left[\mathrm{n}_{1}, \mathrm{n}_{2}, \mathrm{n}_{3}, \mathrm{n}_{4}\right] \Leftrightarrow \mathrm{k}_{\mathrm{i}}-\mathrm{n}_{\mathrm{i}}=0 \text { for } \mathrm{i}=1,2,3,4 .}
\end{aligned}
$$

Besides, from types of points on lines, it is clear that a point and a line of same type is not connected (near). Moreover, the result is equivalent to $\mathrm{T}(\mathrm{X}, \mathrm{Y}) \neq 0$ for a point (or line) $\mathrm{X}$ and a line $\mathrm{Y}$ (or point), respectively. In the other cases, we say that they are connected (near). 


\section{THE MAIN RESULTS}

Now, we will investigate the intersection points of lines in the space $\mathbf{P}\left(\mathbf{J}^{\prime}\right)$.

First we take the different types of lines:

$$
\begin{aligned}
& {[1,0,0,0] \cap[\mathrm{r}, 1,0,0]=\{(0,0,1, \mathrm{z}) \mid \mathrm{z} \in \mathrm{Q}\} \cup\{(0,0,0,1)\},} \\
& {[1,0,0,0] \cap[\mathrm{r}, \mathrm{s}, 1,0]=\{(0,1, \mathrm{~s}, \mathrm{z}) \mid \mathrm{z} \in \mathrm{Q}\} \cup\{(0,0,0,1)\},} \\
& {[1,0,0,0] \cap[\mathrm{r}, \mathrm{s}, \mathrm{t}, 1]=\{(0,1, \mathrm{y}, \mathrm{s}+\mathrm{ty}) \mid \mathrm{y} \in \mathrm{Q}\} \cup\{(0,0,1, \mathrm{t})\},} \\
& {[\mathrm{m}, 1,0,0] \cap[\mathrm{r}, \mathrm{s}, 1,0]=\{(1, \mathrm{~m}, \mathrm{r}+\mathrm{sm}, \mathrm{z}) \mid \mathrm{z} \in \mathrm{Q}\} \cup\{(0,0,0,1)\},} \\
& {[\mathrm{m}, 1,0,0] \cap[\mathrm{r}, \mathrm{s}, \mathrm{t}, 1]=\{(1, \mathrm{~m}, \mathrm{y}, \mathrm{r}+\mathrm{sm}+\mathrm{ty}) \mid \mathrm{y} \in \mathrm{Q}\} \cup\{(0,0,1, \mathrm{t})\},} \\
& {[\mathrm{m}, \mathrm{n}, 1,0] \cap[\mathrm{r}, \mathrm{s}, \mathrm{t}, 1]=\{(1, \mathrm{x}, \mathrm{m}+\mathrm{nx}, \mathrm{r}+\mathrm{sx}+\mathrm{t}(\mathrm{m}+\mathrm{nx}) \mid \mathrm{x} \in \mathrm{Q}\} \cup\{(0,1, \mathrm{n}, \mathrm{s}+\mathrm{tn})\} .}
\end{aligned}
$$

Now we examine the same types of lines:

First we take lines $[\mathrm{m}, \mathrm{n}, \mathrm{p}, 1]$ and $[\mathrm{r}, \mathrm{s}, \mathrm{t}, 1]$ of the fourth types. We can determine the intersection points of these lines in three cases as follows:

i. If $\mathrm{n}-\mathrm{s} \neq 0, \mathrm{p}-\mathrm{t}=0$, then the intersection points are

$$
\left\{\left(1,-(n-s)^{-1}(m-r), y, m-n(n-s)^{-1}(m-r)+p y\right) \mid y \in Q\right\} \cup\{(0,0,1, p=t)\} .
$$

ii. If $\mathrm{p}-\mathrm{t} \neq 0$, then the intersection points are

$$
\begin{aligned}
& \left\{\left(1, x,-(p-t)^{-1}((m-r)+(n-s) x), m+n x-p(p-t)^{-1}((m-r)+(n-s) x)\right) \mid x \in Q\right\} \\
& U\left\{\left(0,1,-(p-t)^{-1}(n-s), n-p(p-t)^{-1}(n-s)\right)\right\} .
\end{aligned}
$$

iii. If $\mathrm{n}-\mathrm{s}=0, \mathrm{p}-\mathrm{t}=0$ and $\mathrm{m}-\mathrm{r} \neq 0$, then the intersection points are

$$
\{(0,1, v, n+p v) \mid v \in Q\} \cup\{(0,0,1, p)\} .
$$

Now we take lines $[\mathrm{m}, \mathrm{n}, 1,0]$ and $[\mathrm{r}, \mathrm{s}, 1,0]$ of the third types. We can determine the intersection points of these lines in two cases as follows:

i. If $\mathrm{m}-\mathrm{r} \neq 0$ and $\mathrm{n}-\mathrm{s}=0$, then the intersection points are

$$
\{(0,1, \mathrm{n}=\mathrm{s}, \mathrm{w}) \mid \mathrm{w} \in \mathrm{Q}\} \cup\{(0,0,0,1)\} .
$$

ii. If $\mathrm{n}-\mathrm{s} \neq 0$, then the intersection points are

$$
\left\{\left(1,-(n-s)^{-1}(m-r), m-n(n-s)^{-1}(m-r), z\right) \mid z \in Q\right\} \cup\{(0,0,0,1)\} .
$$

Finally we take $[\mathrm{m}, 1,0,0]$ and $[\mathrm{r}, 1,0,0]$ of the second types lines. In this case, If $\mathrm{m}-\mathrm{r} \neq 0$, then the intersection points are

$$
\{(0,0,1, \mathrm{w}) \mid \mathrm{w} \in \mathrm{Q}\} \cup\{(0,0,0,1)\} .
$$

Now, conversely, we would like to determine the lines joining these two points, according to the two points in the space $\mathbf{P}\left(\mathbf{J}^{\prime}\right)$ are of different and of the same types. 
First let us examine the case that the two points are of different types:

$$
\begin{aligned}
& (1, \mathrm{x}, \mathrm{y}, \mathrm{z}) \cup(0,1, \mathrm{v}, \mathrm{w})=\{[\mathrm{z}-(\mathrm{w}-\mathrm{tv}) \mathrm{x}-\mathrm{ty}, \mathrm{w}-\mathrm{tv}, \mathrm{t}, 1] \mid \mathrm{t} \in \mathrm{Q}\} \cup\{[\mathrm{y}-\mathrm{vx}, \mathrm{v}, 1,0]\} \\
& (1, \mathrm{x}, \mathrm{y}, \mathrm{z}) \cup(0,0,1, \mathrm{w})=\{[\mathrm{z}-\mathrm{sx}-\mathrm{wy}, \mathrm{s}, \mathrm{w}, 1] \mid \mathrm{s} \in \mathrm{Q}\} \cup\{[\mathrm{x}, 1,0,0]\}, \\
& (1, \mathrm{x}, \mathrm{y}, \mathrm{z}) \cup(0,0,0,1)=\{[\mathrm{y}-\mathrm{sx}, \mathrm{s}, 1,0] \mid \mathrm{s} \in \mathrm{Q}\} \cup\{[\mathrm{x}, 1,0,0]\} \\
& (0,1, \mathrm{y}, \mathrm{z}) \cup(0,0,1, \mathrm{w})=\{[\mathrm{r}, \mathrm{z}-\mathrm{wy}, \mathrm{w}, 1] \mid \mathrm{r} \in \mathrm{Q}\} \cup\{[1,0,0,0]\} \\
& (0,1, \mathrm{y}, \mathrm{z}) \cup(0,0,0,1)=\{[\mathrm{r}, \mathrm{y}, 1,0] \mid \mathrm{r} \in \mathrm{Q}\} \cup\{[1,0,0,0]\} \\
& (0,0,1, \mathrm{z}) \cup(0,0,0,1)=\{[\mathrm{r}, 1,0,0] \mid \mathrm{r} \in \mathrm{Q}\} \cup\{[1,0,0,0]\}
\end{aligned}
$$

Now we can examine the other case. Let the two points be of the same type:

If we take points $(1, \mathrm{x}, \mathrm{y}, \mathrm{z})$ and $(1, \mathrm{u}, \mathrm{v}, \mathrm{w})$ of the first type, then we complete this examination in the following three cases:

i. If $\mathrm{x}-\mathrm{u} \neq 0$, then the lines joining these points are

$$
\begin{aligned}
& \left\{\left[z-((z-w)+c(v-y))(x-u)^{-1} x-c y,(z-w)(x-u)^{-1}+c(v-y)(x-u)^{-1}, c, 1\right] \mid c \in Q\right\} \\
& U\left\{\left[y-(y-v)(x-u)^{-1} x,(y-v)(x-u)^{-1}, 1,0\right]\right\} .
\end{aligned}
$$

ii. If $\mathrm{x}-\mathrm{u}=0, \mathrm{y}-\mathrm{v}=0$ and $\mathrm{z}-\mathrm{w} \neq 0$, then the lines joining these points are

$$
\{[y-s x, s, 1,0] \mid s \in Q\} \cup\{[x, 1,0,0]\} .
$$

iii. If $\mathrm{x}-\mathrm{u}=0$ and $\mathrm{y}-\mathrm{v} \neq 0$, then the lines joining these points are

$$
\left\{\left[z-b x-(z-w)(y-v)^{-1} y, b,(z-w)(y-v)^{-1}, 1\right] \mid b \in Q\right\} \cup\{[x=u, 1,0,0]\} .
$$

If we take points $(0,1, \mathrm{y}, \mathrm{z})$ and $(0,1, \mathrm{v}, \mathrm{w})$ of the second type, then we can determine the lines joining these points in the following two cases:

i. If $y-v \neq 0$, then the lines joining these points are

$$
\left\{\left[a, z-(z-w)(y-v)^{-1} y,(z-w)(y-v)^{-1}, 1\right] \mid a \in Q\right\} \cup\{[1,0,0,0]\} .
$$

ii. If $\mathrm{y}-\mathrm{v}=0$ and $\mathrm{z}-\mathrm{w} \neq 0$, then the lines joining these points are

$$
\{[\mathrm{r}, \mathrm{y}=\mathrm{v}, 1,0] \mid \mathrm{r} \in \mathrm{Q}\} \cup\{[1,0,0,0]\} .
$$

Finally, if we take points $(0,0,1, \mathrm{z})$ and $(0,0,1, \mathrm{w})$ of the third type then we obtain the lines joining these points in the following one case:

i. If $\mathrm{z}-\mathrm{w} \neq 0$, then the lines joining these points are

$$
\{[r, 1,0,0] \mid r \in Q\} \cup\{[1,0,0,0]\} \text {. }
$$

Now, we would like to carry the results over the dual $\operatorname{ring} \mathbf{Q}:=\mathrm{Q}+\mathrm{Q} \varepsilon, \varepsilon \notin \mathrm{Q}$ and $\varepsilon^{2}=0$ with the maximal ideal $\mathbf{I}=\mathrm{Q} \varepsilon$ (of non-units). Note that $\mathbf{Q}$ does not have to be a local ring with the maximal ideal $\mathbf{I}$ in the case $\varepsilon^{2}=\mathrm{k} \in \mathrm{F}-\{0\}$. For, in this case, the inverse of any $\mathrm{x}=\mathrm{a}+\mathrm{b} \varepsilon \in \mathbf{Q}$ would be $\mathrm{x}^{-1}=\mathrm{b}^{-1}\left[\mathrm{a}(\mathrm{ab}-\mathrm{a}-\mathrm{kb})^{-1}\right]+(\mathrm{kb}-$ $\left.a b^{-1} a\right)^{-1} \varepsilon$. As for the case we study, that is, for $k=0$, it is clear that $(a+b \varepsilon)^{-1}=a^{-1}-a^{-1} b a^{-1} \varepsilon$ (we know that 
$\mathrm{a}^{-1}$ exists for all $\left.\mathrm{a} \in \mathbf{Q}-\{0\}\right)$. Therefore, the non-unit elements of $\mathbf{Q}$ consist of the maximal ideal $\mathbf{I}=\mathrm{Q} \varepsilon$. For more detailed information about $\mathbf{Q}$ it can be seen to [3,4]. So, we can find the intersection points of any two lines and the lines joining any two points in the space $\mathbf{P}\left(\mathbf{J}^{\prime \prime}\right)$ where $\mathbf{J}^{\prime \prime}=\mathbf{H}\left(\mathbf{Q}_{4}, \mathrm{~J} \gamma\right)$. By similar calculations, first we take the different types of lines:

$$
\begin{aligned}
{[} & 1, \mathrm{~m}, \mathrm{n}, \mathrm{k}] \cap[\mathrm{q}, 1, \mathrm{~s}, \mathrm{t}] \\
= & \left\{\left((1-\mathrm{mq})^{-1}(\mathrm{~ms}+\mathrm{n}+(\mathrm{mt}+\mathrm{k}) \mathrm{z}), \mathrm{q}(1-\mathrm{mq})^{-1}(\mathrm{~ms}+\mathrm{n}+(\mathrm{mt}+\mathrm{k}) \mathrm{z})+\mathrm{s}+\mathrm{tz}, 1, \mathrm{z}\right) \mid \mathrm{z} \in \mathbf{Q}\right\} \\
& \quad U\left\{\left((1-\mathrm{mq})^{-1}((\mathrm{~ms}+\mathrm{n}) \mathrm{w}+\mathrm{mt}+\mathrm{k}), \mathrm{q}(1-\mathrm{mq})^{-1}((\mathrm{~ms}+\mathrm{n}) \mathrm{w}+\mathrm{mt}+\mathrm{k})+\mathrm{sw}+\mathrm{t}, \mathrm{w}, 1\right) \mid \mathrm{w} \in \mathbf{I}\right\}
\end{aligned}
$$

where $\mathrm{m}, \mathrm{n}, \mathrm{k}, \mathrm{s}, \mathrm{t} \in \mathbf{I}$,

$$
\begin{aligned}
& {[1, \mathrm{~m}, \mathrm{n}, \mathrm{k}] \cap[\mathrm{q}, \mathrm{s}, 1, \mathrm{t}]} \\
& =\left\{\left(\mathrm{m}+\mathrm{n}(1-\mathrm{qn})^{-1}(\mathrm{qm}+\mathrm{s}+(\mathrm{qk}+\mathrm{t}) \mathrm{z})+\mathrm{kz}, 1,(1-\mathrm{qn})^{-1}(\mathrm{qm}+\mathrm{s}+(\mathrm{qk}+\mathrm{t}) \mathrm{z}), \mathrm{z}\right) \mid \mathrm{z} \in \mathbf{Q}\right\} \\
& \mathrm{U}\left\{\left(\mathrm{mv}+\mathrm{n}(1-\mathrm{qn})^{-1}((\mathrm{qm}+\mathrm{s}) \mathrm{v}+\mathrm{qk}+\mathrm{t})+\mathrm{k}, \mathrm{v},(1-\mathrm{qn})^{-1}((\mathrm{qm}+\mathrm{s}) \mathrm{v}+\mathrm{qk}+\mathrm{t}), 1\right) \mid \mathrm{v} \in \mathbf{I}\right\}
\end{aligned}
$$

where $\mathrm{m}, \mathrm{n}, \mathrm{k}, \mathrm{t} \in \mathbf{I}$,

$$
\begin{aligned}
& {[1, \mathrm{~m}, \mathrm{n}, \mathrm{k}] \cap[\mathrm{q}, \mathrm{s}, \mathrm{t}, 1] } \\
&=\left\{\left((1-\mathrm{kq})^{-1}(\mathrm{~m}+\mathrm{ks}+(\mathrm{n}+\mathrm{kt}) \mathrm{y}), 1, \mathrm{y}, \mathrm{q}(1-\mathrm{kq})^{-1}(\mathrm{~m}+\mathrm{ks}+(\mathrm{n}+\mathrm{kt}) \mathrm{y})+\mathrm{s}+\mathrm{ty}\right) \mid \mathrm{y} \in \mathbf{Q}\right\} \\
& \quad \cup\left\{\left((1-\mathrm{kq})^{-1}(\mathrm{n}+\mathrm{kt}+(\mathrm{m}+\mathrm{ks}) \mathrm{v}), \mathrm{v}, 1, \mathrm{q}(1-\mathrm{kq})^{-1}(\mathrm{n}+\mathrm{kt}+(\mathrm{m}+\mathrm{ks}) \mathrm{v})+\mathrm{sv}+\mathrm{t}\right) \mid \mathrm{v} \in \mathbf{I}\right\}
\end{aligned}
$$

where $\mathrm{m}, \mathrm{n}, \mathrm{k} \in \mathbf{I}$,

$$
\begin{aligned}
& {[\mathrm{m}, 1, \mathrm{n}, \mathrm{k}] \cap[\mathrm{q}, \mathrm{s}, 1, \mathrm{t}]} \\
& =\left\{\left(1, \mathrm{~m}+\mathrm{n}(1-\mathrm{sn})^{-1}((\mathrm{q}+\mathrm{sm})+(\mathrm{sk}+\mathrm{t}) \mathrm{z})+\mathrm{kz},(1-\mathrm{sn})^{-1}((\mathrm{q}+\mathrm{sm})+(\mathrm{sk}+\mathrm{t}) \mathrm{z}), \mathrm{z}\right) \mid \mathrm{z} \in \mathbf{Q}\right\} \\
& \quad \mathrm{U}\left\{\left(\mathrm{u},(1-\mathrm{ns})^{-1}((\mathrm{~m}+\mathrm{nq}) \mathrm{u}+\mathrm{nt}+\mathrm{k}), \mathrm{qu}+\mathrm{s}(1-\mathrm{ns})^{-1}((\mathrm{~m}+\mathrm{nq}) \mathrm{u}+\mathrm{nt}+\mathrm{k})+\mathrm{t}, 1\right) \mid \mathrm{u} \in \mathbf{I}\right\}
\end{aligned}
$$

where $\mathrm{n}, \mathrm{k}, \mathrm{t} \in \mathbf{I}$,

$$
\begin{aligned}
& {[\mathrm{m}, 1, \mathrm{n}, \mathrm{k}] \cap[\mathrm{q}, \mathrm{s}, \mathrm{t}, 1]} \\
& =\left\{\left(1,(1-\mathrm{ks})^{-1}((\mathrm{~m}+\mathrm{kq})+(\mathrm{n}+\mathrm{kt}) \mathrm{y}), \mathrm{y}, \mathrm{q}+\mathrm{s}(1-\mathrm{ks})^{-1}((\mathrm{~m}+\mathrm{kq})+(\mathrm{n}+\mathrm{kt}) \mathrm{y})+\mathrm{ty}\right) \mid \mathrm{y} \in \mathbf{Q}\right\} \\
& \quad \mathrm{U}\left\{\left(\mathrm{u},(1-\mathrm{ks})^{-1}((\mathrm{n}+\mathrm{kt})+(\mathrm{m}+\mathrm{kq}) \mathrm{u}), 1, \mathrm{qu}+\mathrm{s}(1-\mathrm{ks})^{-1}((\mathrm{n}+\mathrm{kt})+(\mathrm{m}+\mathrm{kq}) \mathrm{u})+\mathrm{t}\right) \mid \mathrm{u} \in \mathbf{I}\right\}
\end{aligned}
$$

where $\mathrm{n}, \mathrm{k} \in \mathbf{I}$,

$$
\begin{aligned}
& {[\mathrm{m}, \mathrm{n}, 1, \mathrm{k}] \cap[\mathrm{q}, \mathrm{s}, \mathrm{t}, 1]} \\
& =\left\{\left(1, \mathrm{x},(1-\mathrm{kt})^{-1}((\mathrm{~m}+\mathrm{kq})+(\mathrm{n}+\mathrm{ks}) \mathrm{x}), \mathrm{q}+\mathrm{sx}+\mathrm{t}(1-\mathrm{kt})^{-1}((\mathrm{~m}+\mathrm{kq})+(\mathrm{n}+\mathrm{ks}) \mathrm{x})\right) \mid \mathrm{x} \in \mathbf{Q}\right\} \\
& \quad \mathrm{U}\left\{\left(\mathrm{u}, 1,(1-\mathrm{kt})^{-1}((\mathrm{n}+\mathrm{ks})+(\mathrm{m}+\mathrm{kq}) \mathrm{u}), \mathrm{qu}+\mathrm{s}+\mathrm{t}(1-\mathrm{kt})^{-1}((\mathrm{n}+\mathrm{ks})+(\mathrm{m}+\mathrm{kq}) \mathrm{u})\right) \mid \mathrm{u} \in \mathbf{I}\right\}
\end{aligned}
$$

where $\mathrm{k} \in \mathbf{I}$.

Now we examine the same types of lines: 
First we take lines $[\mathrm{m}, \mathrm{n}, \mathrm{k}, 1]$ and $[\mathrm{q}, \mathrm{s}, \mathrm{t}, 1]$ of the fourth types. We can determine the intersection points of these lines in three cases as follows:

i. If $\mathrm{n}$-s $\notin \mathbf{I}, \mathrm{k}-\mathrm{t} \in \mathbf{I}$, then the intersection points are

$$
\begin{aligned}
& \left\{\left(1,-(n-s)^{-1}(m-q+(k-t) y), y, m-n(n-s)^{-1}(m-q+(k-t) y)+k y\right) \mid y \in \mathbf{Q}\right\} \\
& \cup\left\{\left(a,-(n-s)^{-1}(k-t+(m-q) a), 1, m a-n(n-s)^{-1}(k-t+(m-q) a)+k\right) \mid a \in \mathbf{I}\right\} .
\end{aligned}
$$

ii. If $\mathrm{k}-\mathrm{t} \notin \mathbf{I}$, then the intersection points are

$$
\begin{aligned}
& \left\{\left(1, x,-(k-t)^{-1}(m-q+(n-s) x), m+n x-k(k-t)^{-1}(m-q+(n-s) x)\right) \mid x \in \mathbf{Q}\right\} \\
& U\left\{\left(u, 1,-(k-t)^{-1}((m-q) u+n-s), m u+n-k(k-t)^{-1}((m-q) u+n-s)\right) \mid u \in \mathbf{I}\right\} .
\end{aligned}
$$

iii. If $\mathrm{n}-\mathrm{s} \in \mathbf{I}, \mathrm{k}-\mathrm{t} \in \mathbf{I}$ and $\mathrm{m}-\mathrm{q} \notin \mathbf{I}$, then the intersection points are

$$
\begin{aligned}
& \left\{\left(-(m-q)^{-1}(n-s+(k-t) v), 1, v,-m(m-q)^{-1}(n-s+(k-t) v)+n+k v\right) \mid v \in \mathbf{Q}\right\} \\
& \cup\left\{\left(-(m-q)^{-1}((n-s) b+k-t), b, 1,-m(m-q)^{-1}((n-s) b+k-t)+n b+k\right) \mid b \in \mathbf{I}\right\} .
\end{aligned}
$$

Now we take lines $[\mathrm{m}, \mathrm{n}, 1, \mathrm{k}]$ and $[\mathrm{q}, \mathrm{s}, 1, \mathrm{t}]$ of the third types, where $\mathrm{k}, \mathrm{t} \in \mathbf{I}$. We can determine the intersection points of these lines in two cases as follows:

i. If $\mathrm{m}-\mathrm{q} \notin \mathbf{I}$ and $\mathrm{n}-\mathrm{s} \in \mathbf{I}$, then the intersection points are

$$
\begin{aligned}
& \left\{\left(-(m-q)^{-1}(n-s+(k-t) w), 1,-m(m-q)^{-1}(n-s+(k-t) w)+n+k w, w\right) \mid w \in \mathbf{Q}\right\} \\
& \cup\left\{\left(-(m-q)^{-1}((n-s) b+k-t), b,-m(m-q)^{-1}((n-s) b+k-t)+n b+k, 1\right) \mid b \in \mathbf{I}\right\} .
\end{aligned}
$$

ii. If $\mathrm{n}$-s $\notin \mathbf{I}$, then the intersection points are

$$
\begin{aligned}
& \left\{\left(1,-(n-s)^{-1}(m-q+(k-t) z), m-n(n-s)^{-1}(m-q+(k-t) z)+k z, z\right) \mid z \in \mathbf{Q}\right\} \\
& U\left\{\left(a,-(n-s)^{-1}(k-t+(m-q) a), m a-n(n-s)^{-1}(k-t+(m-q) a)+k, 1\right) \mid a \in \mathbf{I}\right\} .
\end{aligned}
$$

Finally we take $[\mathrm{m}, 1, \mathrm{n}, \mathrm{k}]$ and $[\mathrm{q}, 1, \mathrm{~s}, \mathrm{t}]$ of the second types lines, where $\mathrm{n}, \mathrm{k}, \mathrm{s}, \mathrm{t} \in \mathbf{I}$. In this case, if $\mathrm{m}-\mathrm{q} \notin \mathbf{I}$ then the intersection points are

$$
\begin{aligned}
& \left\{\left(-(m-q)^{-1}(n-s+(k-t) w),-m(m-q)^{-1}(n-s+(k-t) w)+n+k w, 1, w\right) \mid w \in \mathbf{Q}\right\} \\
& \cup\left\{\left(-(m-q)^{-1}((n-s) c+k-t),-m(m-q)^{-1}((n-s) c+k-t)+n c+k, c, 1\right) \mid c \in \mathbf{I}\right\} .
\end{aligned}
$$

Now, conversely, we would like to determine the lines joining these two points, according to the two points in the 3 -space are of different and of the same types.

First let us examine the case that the two points are of different types:

$$
\begin{aligned}
& (1, \mathrm{x}, \mathrm{y}, \mathrm{z}) \cup(\mathrm{u}, 1, \mathrm{v}, \mathrm{w}) \\
& =\left\{\left[\mathrm{z}-(\mathrm{w}-\mathrm{zu}-\mathrm{t}(\mathrm{v}-\mathrm{yu}))(1-\mathrm{xu})^{-1} \mathrm{x}-\mathrm{ty},(\mathrm{w}-\mathrm{zu}-\mathrm{t}(\mathrm{v}-\mathrm{yu}))(1-\mathrm{xu})^{-1}, \mathrm{t}, 1\right] \mid \mathrm{t} \in \mathbf{Q}\right\} \\
& \quad \cup\left\{\left[\mathrm{y}-(\mathrm{v}-\mathrm{yu}-\mathrm{k}(\mathrm{w}-\mathrm{zu}))(1-\mathrm{xu})^{-1} \mathrm{x}-\mathrm{kz},(\mathrm{v}-\mathrm{yu}-\mathrm{k}(\mathrm{w}-\mathrm{zu}))(1-\mathrm{xu})^{-1}, 1, \mathrm{k}\right] \mid \mathrm{k} \in \mathbf{I}\right\}
\end{aligned}
$$

where $\mathbf{u} \in \mathbf{I}$, 


$$
\begin{aligned}
& (1, \mathrm{x}, \mathrm{y}, \mathrm{z}) \cup(\mathrm{u}, \mathrm{v}, 1, \mathrm{w}) \\
= & \left\{\left[\mathrm{z}-\mathrm{sx}-(\mathrm{w}-\mathrm{zu}-\mathrm{s}(\mathrm{v}-\mathrm{xu}))(1-\mathrm{yu})^{-1} \mathrm{y}, \mathrm{s},(\mathrm{w}-\mathrm{zu}-\mathrm{s}(\mathrm{v}-\mathrm{xu}))(1-\mathrm{yu})^{-1}, 1\right] \mid \mathrm{s} \in \mathbf{Q}\right\} \\
& \cup\left\{\left[\mathrm{x}-(\mathrm{v}-\mathrm{xu}-\mathrm{k}(\mathrm{w}-\mathrm{zu}))(1-\mathrm{yu})^{-1} \mathrm{y}-\mathrm{kz}, 1,(\mathrm{v}-\mathrm{xu}-\mathrm{k}(\mathrm{w}-\mathrm{zu}))(1-\mathrm{yu})^{-1}, \mathrm{k}\right] \mid \mathrm{k} \in \mathbf{I}\right\}
\end{aligned}
$$

where $\mathrm{u}, \mathrm{v} \in \mathbf{I}$,

$$
\begin{aligned}
& (1, \mathrm{x}, \mathrm{y}, \mathrm{z}) \cup(\mathrm{u}, \mathrm{v}, \mathrm{w}, 1) \\
= & \left\{\left[\mathrm{y}-\mathrm{sx}-(\mathrm{w}-\mathrm{yu}-\mathrm{s}(\mathrm{v}-\mathrm{xu}))(1-\mathrm{zu})^{-1} \mathrm{z}, \mathrm{s}, 1,(\mathrm{w}-\mathrm{yu}-\mathrm{s}(\mathrm{v}-\mathrm{xu}))(1-\mathrm{zu})^{-1}\right] \mid \mathrm{s} \in \mathbf{Q}\right\} \\
& \cup\left\{\left[\mathrm{x}-\mathrm{ny}-(\mathrm{v}-\mathrm{xu}-\mathrm{n}(\mathrm{w}-\mathrm{yu}))(1-\mathrm{zu})^{-1} \mathrm{z}, 1, \mathrm{n},(\mathrm{v}-\mathrm{xu}-\mathrm{n}(\mathrm{w}-\mathrm{yu}))(1-\mathrm{zu})^{-1}\right] \mid \mathrm{n} \in \mathbf{I}\right\}
\end{aligned}
$$

where $\mathrm{u}, \mathrm{v}, \mathrm{w} \in \mathbf{I}$,

$$
\begin{aligned}
& (\mathrm{x}, 1, \mathrm{y}, \mathrm{z}) \cup(\mathrm{u}, \mathrm{v}, 1, \mathrm{w}) \\
= & \left\{\left[\mathrm{q}, \mathrm{z}-\mathrm{qx}-(\mathrm{w}-\mathrm{zv}-\mathrm{q}(\mathrm{u}-\mathrm{xv}))(1-\mathrm{yv})^{-1} \mathrm{y},(\mathrm{w}-\mathrm{zv}-\mathrm{q}(\mathrm{u}-\mathrm{xv}))(1-\mathrm{yv})^{-1}, 1\right] \mid \mathrm{q} \in \mathbf{Q}\right\} \\
& \quad \mathrm{U}\left\{\left[1, \mathrm{x}-(\mathrm{u}-\mathrm{xv}-\mathrm{k}(\mathrm{w}-\mathrm{zv}))(1-\mathrm{yv})^{-1} \mathrm{y}-\mathrm{kz},(\mathrm{u}-\mathrm{xv}-\mathrm{k}(\mathrm{w}-\mathrm{zv}))(1-\mathrm{yv})^{-1}, \mathrm{k}\right] \mid \mathrm{k} \in \mathbf{I}\right\}
\end{aligned}
$$

where $\mathbf{x}, \mathrm{u}, \mathrm{v} \in \mathbf{I}$,

$$
\begin{aligned}
&(\mathrm{x}, 1, \mathrm{y}, \mathrm{z}) \cup(\mathrm{u}, \mathrm{v}, \mathrm{w}, 1) \\
&=\left\{\left[\mathrm{q}, \mathrm{y}-\mathrm{qx}-(\mathrm{w}-\mathrm{yv}-\mathrm{q}(\mathrm{u}-\mathrm{xv}))(1-\mathrm{zv})^{-1} \mathrm{z}, 1,(\mathrm{w}-\mathrm{yv}-\mathrm{q}(\mathrm{u}-\mathrm{xv}))(1-\mathrm{zv})^{-1}\right] \mid \mathrm{q} \in \mathbf{Q}\right\} \\
& \quad \cup\left\{\left[1, \mathrm{x}-\mathrm{ny}-(\mathrm{u}-\mathrm{xv}-\mathrm{n}(\mathrm{w}-\mathrm{yv}))(1-\mathrm{zv})^{-1} \mathrm{z}, \mathrm{n},(\mathrm{u}-\mathrm{xv}-\mathrm{n}(\mathrm{w}-\mathrm{yv}))(1-\mathrm{zv})^{-1}\right] \mid \mathrm{n} \in \mathbf{I}\right\}
\end{aligned}
$$

where $\mathrm{x}, \mathrm{u}, \mathrm{v}, \mathrm{w} \in \mathbf{I}$,

$$
\begin{aligned}
&(\mathrm{x}, \mathrm{y}, 1, \mathrm{z}) \cup(\mathrm{u}, \mathrm{v}, \mathrm{w}, 1) \\
&=\left\{\left[\mathrm{q}, 1, \mathrm{y}-\mathrm{qx}-(\mathrm{v}-\mathrm{yw}-\mathrm{q}(\mathrm{u}-\mathrm{xw}))(1-\mathrm{zw})^{-1} \mathrm{z},(\mathrm{v}-\mathrm{yw}-\mathrm{q}(\mathrm{u}-\mathrm{xw}))(1-\mathrm{zw})^{-1}\right] \mid \mathrm{q} \in \mathbf{Q}\right\} \\
& \cup\left\{\left[1, \mathrm{~m}, \mathrm{x}-\mathrm{my}-(\mathrm{u}-\mathrm{xw}-\mathrm{m}(\mathrm{v}-\mathrm{yw}))(1-\mathrm{zw})^{-1} \mathrm{z},(\mathrm{u}-\mathrm{xw}-\mathrm{m}(\mathrm{v}-\mathrm{yw}))(1-\mathrm{zw})^{-1}\right] \mid \mathrm{m} \in \mathbf{I}\right\}
\end{aligned}
$$

where $\mathrm{x}, \mathrm{y}, \mathrm{u}, \mathrm{v}, \mathrm{w} \in \mathbf{I}$.

Now we can examine the other case. Let the two points be of the same type:

If we take points $(1, \mathrm{x}, \mathrm{y}, \mathrm{z})$ and $(1, \mathrm{u}, \mathrm{v}, \mathrm{w})$ of the first type, then we complete this examination in the following three cases:

i. If $\mathrm{x}-\mathrm{u} \notin \mathbf{I}$, then the lines joining these points are

$$
\begin{aligned}
& \left\{\left[z-(z-w-c(y-v))(x-u)^{-1} x-c y,(z-w-c(y-v))(x-u)^{-1}, c, 1\right] \mid c \in \mathbf{Q}\right\} \\
& U\left\{\left[y-(y-v-t(z-w))(x-u)^{-1} x-t z,(y-v-t(z-w))(x-u)^{-1}, 1, t\right] \mid t \in \mathbf{I}\right\} .
\end{aligned}
$$

ii. If $\mathrm{x}-\mathrm{u} \in \mathbf{I}, \mathrm{y}-\mathrm{v} \in \mathbf{I}$ and $\mathrm{z}-\mathrm{w} \notin \mathbf{I}$, then the lines joining these points are

$$
\begin{aligned}
& \left\{\left[y-s x-(y-v-s(x-u))(z-w)^{-1} z, s, 1,(y-v-s(x-u))(z-w)^{-1}\right] \mid s \in \mathbf{Q}\right\} \\
& \cup\left\{\left[x-n y-(x-u-n(y-v))(z-w)^{-1} z, 1, n,(x-u-n(y-v))(z-w)^{-1}\right] \mid n \in \mathbf{I}\right\} .
\end{aligned}
$$


iii. If $\mathrm{x}-\mathrm{u} \in \mathbf{I}, \mathrm{y}-\mathrm{v} \notin \mathbf{I}$, then the lines joining these points are

$$
\begin{aligned}
& \left\{\left[z-b x-(z-w-b(u-x))(y-v)^{-1} y, b,(z-w-b(u-x))(y-v)^{-1}, 1\right] \mid b \in \mathbf{Q}\right\} \\
& \cup\left\{\left[x-(x-u-k(z-w))(y-v)^{-1} y-k z, 1,(x-u-k(z-w))(y-v)^{-1}, k\right] \mid k \in \mathbf{I}\right\} .
\end{aligned}
$$

If we take points $(\mathrm{x}, 1, \mathrm{y}, \mathrm{z})$ and $(\mathrm{u}, 1, \mathrm{v}, \mathrm{w})$ of the second type, where $\mathrm{x}, \mathrm{u} \in \mathbf{I}$, then we can determine the lines joining these points in the following two cases:

i. If $\mathbf{y}-\mathbf{v} \notin \mathbf{I}$, then the lines joining these points are

$$
\begin{aligned}
& \left\{\left[a, z-a x-(z-w-a(x-u))(y-v)^{-1} y,(z-w-a(x-u))(y-v)^{-1}, 1\right] \mid a \in \mathbf{Q}\right\} \\
& U\left\{\left[1, x-(x-u-k(z-w))(y-v)^{-1} y-k z,(x-u-k(z-w))(y-v)^{-1}, k\right] \mid k \in \mathbf{I}\right\} .
\end{aligned}
$$

ii. If $\mathrm{y}-\mathrm{v} \in \mathbf{I}$ and $\mathrm{z}-\mathrm{w} \notin \mathbf{I}$, then the lines joining these points are

$$
\begin{aligned}
& \left\{\left[q, y-q x-(y-v-q(x-u))(z-w)^{-1} z, 1,(y-v-q(x-u))(z-w)^{-1}\right] \mid q \in \mathbf{Q}\right\} \\
& \cup\left\{\left[1, x-n y-(x-u-n(y-v))(z-w)^{-1} z, n,(x-u-n(y-v))(z-w)^{-1}\right] \mid n \in \mathbf{I}\right\} .
\end{aligned}
$$

Finally, if we take points $(\mathrm{x}, \mathrm{y}, 1, \mathrm{z})$ and $(\mathrm{u}, \mathrm{v}, 1, \mathrm{w})$ of the third type, where $\mathrm{x}, \mathrm{y}, \mathrm{u}, \mathrm{v} \in \mathbf{I}$, then we obtain the lines joining these points in the following one case:

i. If $\mathrm{z}-\mathrm{w} \notin \mathbf{I}$, then the lines joining these points are

$$
\begin{aligned}
& \left\{\left[q, 1, y-q x-(y-v-q(x-u))(z-w)^{-1} z,(y-v-q(x-u))(z-w)^{-1}\right] \mid q \in \mathbf{Q}\right\} \\
& \cup\left\{\left[1, m, x-m y-(x-u-m(y-v))(z-w)^{-1} z,(x-u-m(y-v))(z-w)^{-1}\right] \mid m \in \mathbf{I}\right\} .
\end{aligned}
$$

So, we have completed the examination related to find the intersection points of any two lines and the lines joining any two points in the 3-space $\mathbf{P}\left(\mathbf{J}^{\prime \prime}\right)$. Note that the previous results are obtained if we choose $\varepsilon=0$ (in this case, $\mathbf{I}=\{0\}$ ).

Finally, we would like to make an evaluation between quaternion 2-space (plane) and the quaternion 3space. If we follow the way which is similar to the construction given for 3-space, we have the following for the 2-space $(\mathbf{P}, \mathbf{L}, \mid, \sqcup)$ :

The set of points is

$$
\mathbf{P}=\left\{\left(1, \mathrm{x}_{2}, \mathrm{x}_{3}\right) \mid \mathrm{x}_{2}, \mathrm{x}_{3} \in \mathbf{Q}\right\} \cup\left\{\left(\mathrm{x}_{1}, 1, \mathrm{x}_{3}\right) \mid \mathrm{x}_{1} \in \mathbf{I}, \mathrm{x}_{3} \in \mathbf{Q}\right\} \cup\left\{\left(\mathrm{x}_{1}, \mathrm{x}_{2}, 1\right) \mid \mathrm{x}_{1}, \mathrm{x}_{2} \in \mathbf{I}\right\} .
$$

The set of lines is

$$
\mathbf{L}=\left\{\left[1, \mathrm{~m}_{2}, \mathrm{~m}_{3}\right] \mid \mathrm{m}_{2}, \mathrm{~m}_{3} \in \mathbf{I}\right\} \cup\left\{\left[\mathrm{m}_{1}, 1, \mathrm{~m}_{3},\right] \mid \mathrm{m}_{1} \in \mathbf{Q}, \mathrm{m}_{3} \in \mathbf{I}\right\} \cup\left\{\left[\mathrm{m}_{1}, \mathrm{~m}_{2}, 1\right] \mid \mathrm{m}_{1}, \mathrm{~m}_{2} \in \mathbf{Q}\right\} .
$$

The incidence relation " | is

$$
\begin{aligned}
& {\left[1, \mathrm{~m}_{2}, \mathrm{~m}_{3}\right]=\left\{\left(\mathrm{m}_{2}+\mathrm{m}_{3} \mathrm{y}_{3}, 1, \mathrm{y}_{3}\right) \mid \mathrm{y}_{3} \in \mathbf{Q}\right\} \cup\left\{\left(\mathrm{m}_{2} \mathrm{z}_{2}+\mathrm{m}_{3}, \mathrm{z}_{2}, 1\right) \mid \mathrm{z}_{2} \in \mathbf{I}\right\},} \\
& {\left[\mathrm{m}_{1}, 1, \mathrm{~m}_{3}\right]=\left\{\left(1, \mathrm{~m}_{1}+\mathrm{m}_{3} \mathrm{y}_{3}, \mathrm{y}_{3}\right) \mid \mathrm{y}_{3} \in \mathbf{Q}\right\} \cup\left\{\left(\mathrm{z}_{1}, \mathrm{~m}_{1} \mathrm{z}_{1}+\mathrm{m}_{3}, 1\right) \mid \mathrm{z}_{1} \in \mathbf{I}\right\},} \\
& {\left[\mathrm{m}_{1}, \mathrm{~m}_{2}, 1\right]=\left\{\left(1, \mathrm{y}_{2}, \mathrm{~m}_{1}+\mathrm{m}_{2} \mathrm{y}_{2}\right) \mid \mathrm{y}_{2} \in \mathbf{Q}\right\} \cup\left\{\left(\mathrm{z}_{1}, 1, \mathrm{~m}_{1} \mathrm{z}_{1}+\mathrm{m}_{2}\right) \mid \mathrm{z}_{1} \in \mathbf{I}\right\} .}
\end{aligned}
$$

The connection relation " $\sqcup "$ is 


$$
\begin{aligned}
& \mathrm{P}=\left(\mathrm{x}_{1}, \mathrm{x}_{2}, \mathrm{x}_{3}\right) \sqcup\left(\mathrm{y}_{1}, \mathrm{y}_{2}, \mathrm{y}_{3}\right)=\mathrm{Q} \Leftrightarrow \mathrm{x}_{\mathrm{i}}-\mathrm{y}_{\mathrm{i}} \in \mathbf{I}(\mathrm{i}=1,2,3), \forall \mathrm{P}, \mathrm{Q} \in \mathbf{P} ; \\
& \left.\mathrm{g}=\left[\mathrm{m}_{1}, \mathrm{~m}_{2}, \mathrm{~m}_{3}\right] \sqcup\left[\mathrm{p}_{1}, \mathrm{p}_{2}, \mathrm{p}_{3}\right]=\mathrm{h} \Leftrightarrow \mathrm{m}_{\mathrm{i}}-\mathrm{p}_{\mathrm{i}} \in \mathbf{I}(\mathrm{i}=1,2,3)\right), \forall \mathrm{g}, \mathrm{h} \in \mathbf{L} .
\end{aligned}
$$

The 2-space is isomorphic to the projective Klingenberg plane given in [3,4]. In a projective Klingenberg plane, it is well known that two non-connected (non-neighbour in [3,4]) lines meet at a unique point. However, this situation is different in 3-space as two lines with this propery meet at least at two points (see the results at pages 7 and 8 ). This means that 2-space and 3-space are different from each other.

\section{Acknowledgement}

This work was supported by the Research Fund of the University of Uludağ, Project Number: KUAP(F)-2014/50.

\section{REFERENCES}

[1]. Akpinar, A. and Erdogan, F.O., Dual Quaternionic (n-1)-Spaces Defined by Special Jordan Algebras of Dimension 4n ${ }^{2}-2 n, 2018$ (under review)

[2]. Akpinar, A. and Erdogan, F.O., Collineations and Cross-Ratios in Octonion Planes, 2018 (under review)

[3]. Celik, B. and Erdogan, F.O., On Addition and Multiplication of Points in a Certain Class of Projective Klingenberg Planes, Journal of Inequalities and Applications, 230 (2013).

[4]. Celik, B. and Dayioglu, A., The Collineations which Act as Addition and Multiplication on Points in a Certain Class of Projective Klingenberg Planes, Journal of Inequalities and Applications, 193 (2013).

[5]. Faulkner, J.R., Octonion Planes Defined by Quadratic Jordan Algebras, Mem. Amer. Math. Soc., 104 (1970) 1-71.

[6]. Faulkner, J.R., The Role of Nonassociative Algebra in Projective Geometry, Graduate Studies in Mathematics, Vol. 159, Amer. Math. Soc., Providence, R.I., (2014).

[7]. Jacobson, N., Structure and Representations of Jordan Algebras, Colloq. Publ. 39, Amer. Math. Soc., Providence, R.I., (1968).

[8]. Jacobson, N., Basic Algebra I, W. H. Freeman and Company, New York, (1985).

[9]. McCrimmon, K., The Freudenthal-Springer-Tits Constructions of Exceptional Jordan Algebras, Trans. of the Amer. Math. Soc., 139 (1969) 495-510. 